Pensamiento Crítico N. ${ }^{\circ}$ 14, pp. 47-64

\title{
El comportamiento de la agricultura en la crisis internacional. Resultados a fines del $2009^{1}$
}

\author{
Pedro Barrientos Felipa ${ }^{2}$
}

\section{RESUMEN}

El comportamiento de la economía mundial en los últimos años de la primera década del siglo XXI afecta el desarrollo de los sectores de la economía. Uno de esos sectores es la agricultura, la cual, en su parte moderna tiene una orientación a la exportación; la parte tradicional abastece en gran parte el mercado local. La agricultura tiene gran importancia para el país. No solo por lo que produce, sino por la fuerza laboral que ocupa. El artículo busca exponer fuentes de información que permitan identificar cómo la agricultura enfrentó la crisis y cuáles fueron sus consecuencias. En tal sentido, se exponen los comportamientos de los diversos tipos de cultivo, en donde cada uno tiene su propio comportamiento y que exponen resultados positivos, pero hay que seguir haciendo seguimiento a los compradores

1 El artículo trata de dar respuesta a la siguiente pregunta: ¿Cómo se afecto la producción agrícola y cuáles fueron sus efectos inmediatos, considerando la importancia del sector, en el contexto de la crisis internacional que se presentó en el 2009? Lo reciente del tema implica que se están haciendo investigaciones al respecto, por lo que este trabajo es un aporte al respecto.

2 Economista de la Universidad Ricardo Palma, Lima - Perú. Magíster con Concentración en Marketing de la Universidad del Pacífico, Lima - Perú. Candidato a doctor en Administración de Negocios Globales en la Universidad Ricardo Palma. Profesor principal de la Facultad de Ciencias Económicas y Miembro del Instituto de Investigaciones Económicas de la Universidad Nacional Mayor de San Marcos. Correo electrónico: pbarrientosf@economia.unmsm.pe 


\title{
Pedro Barrientos Felipa
}

y ofertantes internacionales. La conclusión inicial expone que la agricultura puede ser afectada por la crisis, pero su efecto no es tan significativo como en otros sectores.

Palabras clave: Agricultura, Perú, crisis, internacional.

\begin{abstract}
The behaviors of the global economy in the last years of the first decade of this century affected the development of sectors of the economy. One such sector is agriculture, which in turn has a modern, export-oriented one; while the traditional one supplies mostly the local market. Agriculture is of great importance for the country. Not only for what it produces, but by the workforce that it employs This article seeks to expose sources of information to identify how the agricultures is facing the crisis and what were the consequences. In this sense, it exposes the performance of the various types of crops, where each has its own behaviour and exhibit positive results, but we must continue to monitor international buyers and bidders. The initial conclusion states that agriculture can be affected by the crisis and exhibits positive results, but its effect is not as significant as can be observed in others sectors.
\end{abstract}

Keywords: Agriculture, Peru, crisis, international.

\section{INTRODUCCIÓN}

El Perú es uno de los países que es afectado por la crisis económica mundial, si consideramos la tasa de crecimiento del año 2008 (9,8\%), pues en el 2009 tuvo un crecimiento del Producto Bruto Interno (PBI) de 0,9\%, siendo importante el comportamiento positivo del sector construcción y la intervención del gasto público. La agricultura en el 2009 crecio 0,9\%. La economía local todavía manifiesta las consecuencias del persistente deterioro de la economía mundial (aunque se menciona que estamos en un período de estabilización) y no hay presiones inflacionarias. La inflación llegó en el 2008 a $0,25 \%$, habiendo sido en el $20096,65 \%$.

Por el lado del comercio internacional, a fines del año 2009 los resultados de exportaciones se redujeron -respecto a 2008- en US\$ 4444 millones tanto por los resultados en los commodities como en los productos no tradicionales. Sin embargo, se espera una recuperación para los años posteriores, ya que se considera una recuperación de la 


\section{El comportamiento de la agricultura en la crisis internacional. Resultados a fines del 2009}

demanda internacional y las perspectivas favorables de los acuerdos internacionales. La importaciones también se redujeron en US\$ 7428 millones. Estos decrecimientos llevan a que la Balanza Comercial tenga un saldo postivo de US\$ 5873 millones superior a los US\$ 3090 del año anterior.

En este escenario de contracción de la economía mundial y sus consecuencias en la demanda actuó la agricultura peruana. Esta realidad lleva a que se realicen acciones que se complementan: las medidas que toma el gobierno nacional para el apoyo a la agricultura y las acciones que deberán realizarse por parte del sector privado o inversionistas. El primer momento está relacionado a la contracción de los compradores, que implica que además de nuestro país hay otros que están siendo afectados y que tienen que vender sus productos de todas maneras. De por sí esto exige medidas relacionadas a los costos, en cuanto a hacerlos que aumenten la productividad (tarea de los inversionistas) y la vez su competitividad como exportadores. Los países que se adecuan a estas circunstancias tendrán ventajas y estarán encima de sus competidores.

El presente artículo, es la primera parte de la investigación que se realizó de acuerdo a los lineamientos del Instituto de Investigaciones Económicas de la Universidad Nacional Mayor de San Marcos. Para su realización se consultó fuentes bibliográficas publicadas en los principales medios locales. En tal sentido, es una investigación exploratoria con base en libros y documentos oficiales. A través de la contrastación de los fundamentos teóricos y empíricos se realizó las inferencias, que arrojan como resultado, las correspondientes explicaciones parciales del fenómeno investigado.

\section{LA CRISIS ECONÓMICA INTERNACIONAL}

Parece haber una coincidencia en los investigadores del tema que el origen de la crisis procede de la fuerte expansión del crédito hipotecario en los Estados Unidos, en un entorno de tipos de interés reducidos en términos nominales y negativos en términos reales. La actual crisis financiera se inicia en EE. UU., respecto al crédito, el consumo y la inversión especulativa. Para la UNCTAD (2009), la crisis financiera mundial se desató en una situación marcada por la incapacidad de la comunidad internacional para dotar

a la economía globalizada de reglas mundiales creíbles, especialmente en lo referente a las relaciones financieras internacionales y las políticas macroeconómicas. Las burbujas 


\section{Pedro Barrientos Felipa}

especulativas, empezando por la burbuja inmobiliaria de los Estados Unidos, fueron el fruto de una política de desregulación activa de los mercados financieros a escala mundial, ampliamente respaldada por gobiernos de todo el mundo.

Un análisis fuertemente crítico a lo que se inicia a finales de la primera década del siglo XXI es lo que manifiesta Arroyo (2009) en cuanto a que

Tras veinte años de haber sido adoctrinados, desde el Consenso de Washington (1990), por las concepciones de que el Estado no participaba en la economía, que la economía corría sola sin ninguna regulación y que todo bien o servicio debía ser privatizado, la crisis del modelo llevó al retorno del Estado y de la política por encima del mercado. El Estado salvó a múltiples empresas prestándoles dinero o nacionalizándolas e inyectó miles de millones de dólares para salvar su sistema financiero. Los evangelios neoliberales cayeron por tierra y el Estado hizo su reaparición necesaria y triunfal en el escenario económico. Hoy se van salvando con injerencia del Estado el City Group y hacen cola para el salvataje financiero los colosos General Motors, Chrysler y Ford, empresas emblemáticas del país del Tío Sam.

En un sentido más amplio, es lo que opina Jacques Ginesta (2009), en cuanto a que

parece equivocado restringir las crisis globales al auge de la globalización propio de las últimas décadas. Sin que las economías estuvieran tan fuertemente conectadas como ahora, es innegable que la crisis financiera norteamericana de 1929 tuvo repercusiones no sólo en los Estados Unidos y Europa, sino en todo el mundo y duramente en América Latina, donde la contracción del crédito, el consumo la producción y las exportaciones, generó una sucesión de golpes de Estado, seguidos de dictaduras más o menos prolongadas en un intento de manejar las tensiones sociales que se generaron. La reducción de los apoyos económicos no permitió a los gobiernos de la época dar respuesta satisfactoria mediante el normal ejercicio de las instituciones al aumento de las demandas sociales, producto de la caída de la actividad económica.

Por primera vez en sesenta años la economía mundial registró una caída en el nivel de actividad en 2009, observándose comportamientos distintos a lo largo del año. El Banco Central de Reserva (2009) manifiesta que "mientras el primer semestre se registró una contracción severa en las economías desarrolladas, una marcada desaceleración en las economías emergentes más dinámicas y el mantenimiento de condiciones 


\section{El comportamiento de la agricultura en la crisis internacional. \\ Resultados a fines del 2009}

desfavorables en los mercados financieros. Hacia el segundo semestre, sin embargo, la actividad económica mundial empezó a mostrar signos de recuperación, principalmente en la actividad manufacturera y el comercio internacional, gracias al efecto de las políticas de estímulo monetario y fiscal". El Cuadro 1 expone los resultados de crecimiento en el 2009, en donde los países desarrollados son los que mayormente han sido afectados con esta crisis.

Cuadro 1. Crecimiento mundial (variaciones porcentuales).

\begin{tabular}{|l|r|r|r|r|r|}
\hline & 2005 & 2006 & \multicolumn{1}{|c|}{2007} & 2008 & \multicolumn{1}{|c|}{2009} \\
\hline Economías desarrolladas & $\mathbf{2 , 6}$ & $\mathbf{3 , 0}$ & $\mathbf{2 , 7}$ & $\mathbf{0 , 5}$ & $-\mathbf{3 , 2}$ \\
\hline 1. Estados Unidos & 3,1 & 2,7 & 2,1 & 0,4 & $-2,4$ \\
\hline 2. Eurozona & 1,8 & 3,1 & 2,8 & 0,6 & $-4,0$ \\
\hline 3. Japón & 1,9 & 2,0 & 2,4 & $-1,2$ & $-5,2$ \\
\hline 4. Reino Unido & 2,2 & 2,9 & 2,6 & 0,5 & $-4,9$ \\
\hline 5. Canadá & 3,0 & 2,9 & 2,5 & 0,4 & $-2,6$ \\
\hline 6. Otras economías desarrolladas & 4,0 & 4,6 & 4,7 & 1,7 & $-1,1$ \\
\hline Economías en desarrollo & $\mathbf{7 , 1}$ & $\mathbf{7 , 9}$ & $\mathbf{8 , 3}$ & $\mathbf{6 , 1}$ & $\mathbf{2 , 4}$ \\
\hline 1. África & 5,7 & 6,1 & 6,3 & 5,5 & 2,1 \\
\hline 2. Europa central y del este & 6,0 & 6,6 & 5,5 & 3,1 & $-3,9$ \\
\hline 3. Comunidad de estados & 6,7 & 8,4 & 8,6 & 5,5 & $-6,6$ \\
\hline independientes & 6,4 & 7,7 & 8,1 & 5,6 & $-7,9$ \\
\hline Rusia & 9,0 & 9,8 & 10,6 & 7,9 & 6,6 \\
\hline 4. Asia en desarrollo & 10,4 & 11,6 & 13,0 & 9,6 & 8,7 \\
\hline China & 9,2 & 9,8 & 9,4 & 7,5 & 5,7 \\
\hline India & 5,5 & 5,8 & 6,2 & 5,1 & 2,4 \\
\hline 5. Medio Este & 4,7 & 5,6 & 5,8 & 4,2 & $-1,8$ \\
\hline 6. América Latina y Caribe & 3,2 & 4,0 & 6,1 & 5,1 & $-0,2$ \\
\hline Brasil & $\mathbf{4 , 5}$ & $\mathbf{5 , 1}$ & $\mathbf{5 , 2}$ & $\mathbf{3 , 0}$ & $\mathbf{- 0 , 6}$ \\
\hline Economía mundial & & & & & \\
\hline Nota: & 8,5 & 9,6 & 10,6 & 8,0 & 4,9 \\
\hline BRIC's 1/ & 4,4 & 4,7 & 4,7 & 2,7 & $-1,1$ \\
\hline Socios comerciales 2/ & & & & \\
\hline
\end{tabular}

1/ Brasil, Rusia, India y China

2/ Canasta de los 20 principales socios comerciales de Perú

Fuente: Banco Central de Reserva - Memoria Institucional 2009 


\section{Pedro Barrientos Felipa}

Los resultados también exponen el comportamiento de los nuevos paises competitivos que para algunos tratadistas la nueva polarización de la economía, en cuanto que el movimiento comercial de los países ya no depende sólo de Estados Unidos o de lo que se realice en Europa Occidental, sino que ahora hay que considerar a China e India, o a los BRIC's. Por lo tanto, esta crisis plantea retos diferentes a los más afectados, comportamientos que no se habían observado en la de inicios del siglo XX (1929), en donde el papel del Estado fue relevante y que hoy se considera -nuevamente-como un agente importante para inyectar dinamismo a las economías locales.

Dentro del actual contexto de globalización, la polarización económica es de extrema importancia tanto para los países desarrollados como para aquellas economías en desarrollo. Es una alternativa para modificar la cartera de exportaciones e importaciones, en donde las variables costos, precios y calidad van a estar mucho más cercanas en la toma de decisiones. La actual situación de polarización tiene su origen en varios factores, pero está claro que a las economías en desarrollo les puede convenir si toman una mayor conciencia de lo que está ocurriendo y cómo aprovecharlo.

La crisis financiera que está viviendo el mundo, en realidad es una crisis económica mundial, que confirma que el mundo comercial ya es diferente, en donde hay países que buscan un mayor protagonismo. El mundo no será igual que antes. Tanto las causas de la crisis, como las consecuencias, son motivo de profundas reflexiones y análisis. El conjunto de soluciones que se aplique, que tiene agudos procesos de reflexión y análisis por parte de los gobiernos involucrados y con mayor poder, confirma el nuevo orden mundial. Se encuentra en el centro de la discusión, precisamente la concepción de Estado y su papel frente a la economía y la sociedad para el siglo XXI. Los gobiernos tienen que sentarse a concertar la nueva forma económica de ver el mundo y la economía.

Los resultados que se muestran, a fines del 2009, explican que la crisis es de tipo estructural (no es coyuntural), que tendrá una estancia de largo plazo en los principales países industrializados. Y que la crisis no solo compromete al sector financiero o a las finanzas internacionales. Al tomar parte de la economía real, la crisis, muestra los principales problemas de los países, especialmente de los que antes se consideraban los motores de la economía mundial. ¿Cómo competir Estados Unidos y la Europa Occidental con las actividades económicas de lo que hace China e India? El efecto se 


\section{El comportamiento de la agricultura en la crisis internacional. Resultados a fines del 2009}

verá en la generación del empleo. La crisis afecta a unos países más que a otros, no todos han sido afectados por igual; algunos por una buena administración política, otros porque no estaban en ese momento pero que si son frágiles, si no toman las medidas que corresponden. Corresponde hacer un análisis de que fue lo que hizo posible que el efecto no generará crisis locales o en cuanto que no originó un paro en la economía. El escenario en los países desarrollados, a estas alturas, se circunscribe a mostrar una desaceleración y un reacomodo en la administración de sus capitales, obligando a utilizar medidas como las siguientes:

- Política monetaria de baja de tasas de interés, tratando de impulsar las solicitudes de crédito por los productores y a partir de ahí generar empleo.

- Políticas fiscales de estímulo a la actividad económica: esencialmente implica una reducción de impuestos y el aumento de la inversión pública.

- Planes de salvataje para entidades financieras.

Los resultados adversos, consecuencia de la crisis mundial, amenazan con borrar los progresos en la agricultura mundial, que ha estado enfrascada en la lucha contra la pobreza. Como explica IICA (2009):

lo que a inicios de 2008 se perfilaba como una tormenta perfecta, con un crecimiento acelerado en los precios de los productos básicos, bajos inventarios mundiales, una demanda creciente impulsada por tres vectores (el gran crecimiento de China e India, la demanda por granos para producir biocombustibles y las inversiones especulativas en los mercados de futuros), ha dado paso al escenario más complicado de una crisis financiera que repercute en el sector real con una recesión económica, cuyos efectos iniciales hacen recordar los aciagos días de la depresión de los años treinta, pero ahora en un mundo más interdependiente y globalizado.

Los precios favorables de los principales commodities agrícolas en los años 2008 y 2009 beneficiaron temporalmente a los países exportadores de dichos productos (Cuadro 2); para el Perú los precios intencionales del azúcar y el café fueron favorables. Sin embargo, los costos de producción de estos se elevaron debido al crecimiento de los precios de los insumos, en particular de los fertilizantes. Como hemos expuesto, los gobiernos de muchos países han enfocado sus esfuerzos en políticas anticíclicas y medidas de corto plazo. La gran mayoría ha relegado la adopción de medidas orientadas a 


\section{Pedro Barrientos Felipa}

posicionar a la agricultura y la economía rural de sus países con una visión de más largo aliento, lo cual explica el riesgo a la cual está expuesto este sector, muy en especial en el caso de los pequeños agricultores.

Cuadro 2. Cotizaciones Internacionales productos agrarios.

\begin{tabular}{|c|c|c|c|c|c|c|c|c|c|}
\hline Año & $\begin{array}{l}\text { Azúcar } \\
\text { Cont.11 } \\
\text { US\$/tm }\end{array}$ & $\begin{array}{l}\text { Azúcar } \\
\text { Cont.14 } \\
\text { US\$/tm }\end{array}$ & $\begin{array}{c}\text { Café } \\
\text { Otr.S.Aráb. } \\
\text { US\$/tm }\end{array}$ & $\begin{array}{c}\text { Trigo } \\
\text { EE.UU. } \\
\text { US\$/tm }\end{array}$ & $\begin{array}{c}\text { Maíz } \\
\text { EE.UU. } \\
\text { US\$/tm }\end{array}$ & $\begin{array}{c}\text { Arroz } \\
\text { Thailand } \\
\text { US\$/tm }\end{array}$ & $\begin{array}{l}\text { Aceite } \\
\text { de soya } \\
\text { EE.UU. } \\
\text { US\$/tm }\end{array}$ & $\begin{array}{c}\text { Frijol } \\
\text { de soya } \\
\text { EE.UU. } \\
\text { US\$/tm }\end{array}$ & $\begin{array}{l}\text { Harina } \\
\text { de soya } \\
\text { EE.UU. } \\
\text { US\$/tm }\end{array}$ \\
\hline 2000 & 187,69 & 420,61 & 1920,36 & 100,64 & 72,55 & 187,73 & 330,69 & 181,18 & 191,27 \\
\hline 2001 & 201,04 & 465,30 & 1372,01 & 106,78 & 74,41 & 169,54 & 317,87 & 170,11 & 186,44 \\
\hline 2002 & 173,60 & 459,46 & 1330,27 & 133,20 & 84,57 & 196,86 & 402,74 & 190,96 & 185,85 \\
\hline 2003 & 165,52 & 471,89 & 1412,42 & 131,95 & 88,73 & 200,34 & 519,39 & 235,99 & 218,24 \\
\hline 2004 & 189,85 & 450,90 & 1770,02 & 134,41 & 93,66 & 244,79 & 629,82 & 279,61 & 260,70 \\
\hline 2005 & 250,60 & 470,03 & 2523,94 & 129,66 & 74,44 & 293,54 & 506,84 & 223,62 & 206,61 \\
\hline 2006 & 342,09 & 488,57 & 2509,88 & 169,12 & 93,91 & 313,01 & 538,83 & 214,14 & 193,94 \\
\hline 2007 & 255,73 & 463,45 & 2717,34 & 231,19 & 137,85 & 337,20 & 768,16 & 306,74 & 253,70 \\
\hline 2008 & 305,17 & 469,01 & 3050,21 & 293,12 & 192,12 & 684,53 & 1092,22 & 447,73 & 367,48 \\
\hline 2009 & 411,61 & 541,45 & 3 128,68 & 192,54 & 139,38 & 561,45 & 728,93 & 384,49 & 383,80 \\
\hline
\end{tabular}

Fuente: Banco Central de Reserva del Perú - Memoria Institucional 2009

Podemos concluir, por lo que sabemos, que la crisis financiera internacional es un detonante y que el escenario es de una crisis económica mundial. Todos los países actores, primarios y secundarios, están siendo afectados o lo serán. El escenario es complejo y representa un reto para los gobernantes en donde no hay cabida para medidas aisladas dentro del país ni dejar de lado las relaciones internacionales comerciales, considerando a la agricultura como un desafío. Las expectativas de muchos deben ser modificadas, buscando nuevas oportunidades, pudiendo países como el Perú obtener provecho comercial. La crisis en su complejidad genera oportunidades o ayuda a identificarlas pues aún estando presentes no se toma en cuenta su presencia. El mundo 


\section{El comportamiento de la agricultura en la crisis internacional. \\ Resultados a fines del 2009}

no es el mismo y la interrelación entre los países ahora es mucho más rápida y menos compleja que antes.

\section{AMÉRICA LATINA FRENTE A LA CRISIS INTERNACIONAL}

Lo expresado explica que la crisis es sistémica y que al parecer tiene efectos de largo plazo. Para América Latina es una brillante oportunidad para aplicar medidas que le permitan romper con algunos paradigmas que afectan su desarrollo que la llevan a considerar que no es posible seguir avanzando si no avanzan los países impulsores de la economía mundial, especialmente Estados Unidos, Europa y Japón. Ahora hay nuevos actores de los cuales se puede aprovechar su crecimiento económico para considerarlos como nuevos impulsores. De ahí que América Latina ha hecho un enorme esfuerzo para consolidar políticas macroeconómicas que le dan estabilidad a las economías. Por tanto,

se enfrenta a la crisis financiera internacional mejor preparada, en general, que en épocas anteriores: bancos centrales con más autonomía, sistemas financieros más estables, deudas externas reducidas, reservas importantes y cuentas fiscales y exteriores con superávit. Además, existe voluntad política de los gobiernos de la región para combatir la inestabilidad económica y la mayoría ya está trabajando en esa dirección (ANEP, 2008).

Los efectos más negativos y generalizados de la crisis mundial sobre la agricultura latinoamericana son resultado de una caída prevista en cerca de $11 \%$ en el volumen del comercio mundial y una drástica contracción de los flujos internacionales de crédito privado y de remesas. En casi todos los países, la combinación de estos efectos está aumentando la vulnerabilidad alimentaria de la población, especialmente en territorios rurales. Si la crisis alimentaria se relacionó originalmente con los precios altos, la crisis financiera está asociada a menores ingresos que, como demuestran las experiencias anteriores, tardan muchos años en recuperarse.

En el Cuadro 3 se expone el crecimiento de los países latinoamericanos que presentó CEPAL en su oportunidad. Tanto lo que se esperaba en el 2009 como en el 2010. En el caso de nuestro país se proyectaba un crecimiento de $2,0 \%$, siendo al final $0.9 \%$. Aunque los resultados son diferentes, entre lo proyectado y lo real, lo cierto es que se creció, resultado que tiene su explicación en el esfuerzo del gasto fiscal, considerando que la inversión privada se contrajo influenciada por la crisis mundial. 


\section{Pedro Barrientos Felipa}

Cuadro 3. Crecimiento del PBI en los países latinoamericanos (variaciones porcentuales).

\begin{tabular}{|l|c|c|c|c|}
\hline & $\mathbf{2 0 0 7}$ & $\mathbf{2 0 0 8}$ & $\mathbf{2 0 0 9} \mathbf{a} /$ & $\mathbf{2 0 1 0} \mathbf{a} /$ \\
\hline Argentina & 8,7 & 7,0 & 1,5 & 3,0 \\
Bolivia & 4,6 & 6,1 & 2,5 & 3,5 \\
Brasil & 5,7 & 5,1 & $-0,8$ & 3,5 \\
Chile & 4,7 & 3,2 & $-1,0$ & 3,5 \\
Colombia & 7,5 & 2,6 & 0,6 & 3,5 \\
Costa Rica & 7,8 & 2,6 & $-3,0$ & 3,0 \\
Cuba & 7,3 & 4,3 & 1,0 & 3,0 \\
Ecuador & 2,5 & 6,5 & 1,0 & 2,5 \\
El Salvador & 4,7 & 2,5 & $-2,0$ & 2,5 \\
Guatemala & 6,3 & 4,0 & $-1,0$ & 2,5 \\
Haití & 3,4 & 1,3 & 2,0 & 2,0 \\
Honduras & 6,3 & 4,0 & $-2,5$ & 2,5 \\
México & 3,3 & 1,3 & $-7,0$ & 2,5 \\
Nicaragua & 3,2 & 3,2 & $-1,0$ & 2,5 \\
Panamá & 11,5 & 9,2 & 2,5 & 5,0 \\
Paraguay & 6,8 & 5,8 & $-3,0$ & 3,0 \\
Perú & 8,9 & 9,8 & 2,0 & 5,0 \\
República Dominicana & 8,5 & 5,3 & 1,0 & 2,0 \\
Uruguay & 7,6 & 8,9 & 1,0 & 3,5 \\
Venezuela & 8,9 & 4,8 & 0,3 & 3,5 \\
Subtotal América Latina & $\mathbf{5 , 8}$ & $\mathbf{4 , 2}$ & $-\mathbf{1 , 9}$ & $\mathbf{3 , 2}$ \\
Caribe & 3,4 & 1,5 & $-1,2$ & 0,5 \\
América Latina y el Caribe & $\mathbf{5 , 8}$ & $\mathbf{4 , 2}$ & $\mathbf{- 1 , 9}$ & $\mathbf{3 , 1}$ \\
\hline
\end{tabular}

a/ Proyección

Fuente: Comisión Económica para América Latina y el Caribe - Cepal. 


\section{El comportamiento de la agricultura en la crisis internacional. Resultados a fines del 2009}

Pero, más allá de las vías de contagio y de los efectos de la recesión mundial en las economías latinoamericanas y la recuperación de algunos precios, parece indudable que la crisis financiera internacional dejará secuelas perdurables en la economía real de algunos países mayor desempleo, menor crecimiento económico, más contracción comercial y déficits fiscales difíciles de superar. Aunque ya se aprecian signos de recuperación, este proceso será lento y gradual. Para tal caso, es un momento para generar una nueva forma de tratar el agro, en el cual el Estado tiene que conciliar con las grandes empresas agrarias en beneficio también de las medianas, pequeñas y microempresas. También el considerar en sus políticas en cómo promover la transformación de la agricultura en una industria con mayor grado de transformación. Para tal caso, se puede partir de las siguientes ideas:

- Establecer nuevos sistemas de asistencia técnica que tome en cuenta la competitividad y las posibilidades de producción, poniendo énfasis en adaptarse a nuevos mercados.

- Crear un sistema de financiamiento para inmiscuir a los medianos y pequeños productores agrícolas con orientación al mercado internacional. Debe permitírseles acceso a una mejor tecnología, en donde los costos financieros deben tener un papel clave.

- Considerar que la producción agraria no está desligada de la seguridad alimentaria de cada uno de los países. En tal sentido, hay que crear una institucionalidad que permita proteger a los ciudadanos ante prácticas adversas.

- Las posibilidades de los inversionistas para que ingresen a nuevos mercados basándose en un mayor grado de transformación de los productos agrícolas y para lo cual el Estado debe estar alerta en la creación de mecanismos que incentiven este siguiente estadio.

\section{EFECTOS DE LA CRISIS INTERNACIONAL EN EL PERÚ}

Para el Perú es el mismo escenario de crisis internacional, las tasas de crecimiento han sido relativamente bajas. Comparando con otros países latinoamericanos ha habi- 


\section{Pedro Barrientos Felipa}

do un comportamiento favorable. El PBI en el año 2009 creció 0,9\%, lo cual explica que de todas maneras la crisis mundial nos afectó como país. El año anterior, 2008, se había logrado una tasa de $9,8 \%$, la tasa de crecimiento más alta de los últimos tiempos. Los sectores económicos que muestran un comportamiento negativo (Cuadro 4) son Pesca, Manufactura y Comercio. El crecimiento de la agricultura tiene una tasa de 0,9\%.

Cuadro 4: Producto Bruto Interno (Variaciones porcentuales)

\begin{tabular}{|l|c|c|c|c|c|}
\hline & $\mathbf{2 0 0 5}$ & $\mathbf{2 0 0 6}$ & $\mathbf{2 0 0 7}$ & $\mathbf{2 0 0 8}$ & $\mathbf{2 0 0 9}$ \\
\hline Agropecuario & 5,4 & 8,4 & 3,2 & 7,2 & 2,3 \\
\hline Agrícola & 4,2 & 8,4 & 2,0 & 7,4 & 0,9 \\
\hline Pecuario & 6,9 & 8,2 & 5,3 & 6,0 & 4,4 \\
\hline Pesca & 3,2 & 2,4 & 6,9 & 6,3 & $-7,9$ \\
\hline Minería e hidrocarburos & 8,4 & 1,4 & 2,7 & 7,6 & 0,6 \\
\hline Minería metálica y no metálica & 7,3 & 1,0 & 2,4 & 7,4 & $-0,9$ \\
\hline Hidrocarburos & 23,4 & 5,7 & 6,5 & 10,3 & 16,1 \\
\hline Manufactura & 7,5 & 7,5 & 11,1 & 9,1 & $-7,2$ \\
\hline De procesamiento de recursos primarios & 3,9 & 4,1 & $-2,7$ & 7,6 & 0,0 \\
\hline No primaria & 8,5 & 8,5 & 14,0 & 8,9 & $-8,5$ \\
\hline Electricidad y agua & 5,6 & 6,9 & 8,5 & 7,8 & 1,2 \\
\hline Construcción & 8,4 & 14,8 & 16,6 & 16,5 & 6,1 \\
\hline Comercio & 6,2 & 11,7 & 9,7 & 13,0 & $-0,4$ \\
\hline Otros servicios & 6,8 & 6,8 & 9,0 & 9,1 & 3,1 \\
\hline PRODUCTO BRUTO INTERNO & $\mathbf{6 , 8}$ & $\mathbf{7 , 7}$ & $\mathbf{8 , 9}$ & $\mathbf{9 , 8}$ & $\mathbf{0 , 9}$ \\
\hline
\end{tabular}

Fuente: Banco Central de Reserva del Perú - Memoria Institucional 2009.

La crisis financiera mundial ha afectado a muchas actividades económicas peruanas y las exportaciones de productos no tradicionales no son ajenas a ello. Sin embargo, dentro de este marco, cabe mencionar que la agricultura peruana fue una de las actividades afectadas por la actual crisis de ahí que expone un pequeño crecimiento. El 


\section{El comportamiento de la agricultura en la crisis internacional. \\ Resultados a fines del 2009}

PBI agrícola (cuadro 5) y su leve crecimiento está explicado por la significativa caída a la agroexportación que tuvo un crecimiento negativo de $10,9 \%$, mientras que el año anterior o sea 2008, había logrado un crecimiento de $14,8 \%$. Entre los productos que declinaron en su producción significativamente por contracción de la demanda en el exterior tenemos: algodón en rama, aceituna, mango, marigold y té. El principal producto que acapara la mayor exportación en la agricultura de exportación, como es el espárrago, tuvo una caída de $4.4 \%$.

Cuadro 5. Producción agrícola por principales productos (Variaciones porcentuales).

\begin{tabular}{|lrrrrr|}
\hline & $\mathbf{2 0 0 5}$ & $\mathbf{2 0 0 6}$ & $\mathbf{2 0 0 7}$ & $\mathbf{2 0 0 8}$ & $\mathbf{2 0 0 9}$ \\
\hline PBI agrícola & $\mathbf{4 . 2}$ & $\mathbf{8 . 4}$ & $\mathbf{2 . 0}$ & $\mathbf{7 . 4}$ & $\mathbf{0 . 9}$ \\
Mercado Interno & 7.5 & 4.6 & 3.0 & 4.3 & 6.2 \\
Agroexportación e Industrial & $\mathbf{- 3 . 8}$ & $\mathbf{1 8 . 5}$ & $\mathbf{- 0 . 5}$ & $\mathbf{1 4 . 8}$ & $\mathbf{- 1 0 . 9}$ \\
Café & -18.5 & 44.9 & -17.3 & 21.1 & -6.9 \\
Caña de azúcar & -9.2 & 14.9 & 13.6 & 14.2 & 7.5 \\
Maíz a. duro & 1.6 & 2.1 & 10.1 & 9.7 & 2.2 \\
Algodón en Rama & 10.5 & 2.9 & 1.0 & -22.3 & -42.7 \\
Espárrago & 7.0 & 26.2 & 9.3 & 15.6 & -4.4 \\
Uva & 9.1 & 13.0 & 2.6 & 13.6 & 18.4 \\
Aceituna & 28.6 & -3.9 & -0.1 & 118.1 & -93.7 \\
Mango & -15.3 & 36.1 & -8.1 & 9.6 & -48.7 \\
Cacao & -2.6 & 24.8 & -0.4 & 7.5 & 6.2 \\
Palta & -4.6 & 9.5 & 7.5 & 12.0 & 14.4 \\
Palma aceitera & -4.1 & 18.2 & 0.9 & 3.3 & 6.2 \\
Marigold & 14.4 & -56.2 & -59.7 & -55.8 & -29.3 \\
Orégano & 14.5 & 8.4 & 17.4 & 33.5 & 19.7 \\
Té & 173.6 & 13.8 & -25.4 & 11.5 & -21.0 \\
\hline
\end{tabular}

Fuente: Banco Central de Reserva del Perú - Memoria Institucional 2009

En lo que corresponde a la agricultura de exportación, en la cartera de países clientes Estados Unidos fue nuestro mejor comprador en el 2009. El 28,3\% de la producción agraria se canalizó hacia este mercado. Este resultado muestra la potencialidad de otros mercados en los cuales recién se está exportando en grandes volúmenes, como puede 


\section{Pedro Barrientos Felipa}

ser el caso de China. Al observar el Cuadro 6, podemos apreciar la ubicación expectante de China como comprador, siendo superado por países cuya potencialidad prácticamente está en su límite, mientras que el país asiático es un reciente socio comercial, lo que da la idea del potencial que se tiene para las exportaciones agrícolas; también hay que considerar a países como Brasil, India, Rusia y otros países asiáticos.

Cuadro 6. Perú - Ranking de las Exportaciones Agrarias 2009.

\begin{tabular}{|l|l|r|r|c|}
\hline Ranking & \multicolumn{1}{|c|}{ País } & $\begin{array}{r}\text { Peso Neto Total } \\
\text { (TM) }\end{array}$ & $\begin{array}{r}\text { Valor FOB (miles } \\
\text { US } \$ \text { ) }\end{array}$ & Participación (\%) \\
\hline 1 & TOTAL & -- & $\mathbf{2 ~ 6 2 7 ~ 7 8 2 , 5}$ & $\mathbf{1 0 0 , 0}$ \\
2 & Estados Unidos & 460239,6 & 743297,7 & 28,3 \\
3 & Holanda & 205318,2 & 266270,7 & 10,1 \\
4 & Alemania & 80106,2 & 228312,7 & 8,7 \\
5 & España & 120816,4 & 217954,6 & 8,3 \\
6 & Colombia & 154086,9 & 128350,7 & 4,9 \\
7 & Ecuador & 144377,8 & 98869,8 & 3,8 \\
8 & Bélgica & 32472,8 & 87401,3 & 3,3 \\
9 & Inglaterra & 44016,9 & 87022,1 & 3,3 \\
10 & Francia & 23130,7 & 64370,8 & 2,5 \\
11 & México & 52590,3 & 63403,7 & 2,4 \\
12 & Italia & 16623,8 & 46062,7 & 1,8 \\
13 & China & 36255,3 & 45879,2 & 1,8 \\
14 & Canadá & 24205,0 & 43375,4 & 1,7 \\
15 & Chile & 59259,7 & 42384,0 & 1,6 \\
16 & Haiti & 43991,9 & 41980,9 & 1,6 \\
17 & Japón & 19780,0 & 37379,5 & 1,4 \\
18 & Bolivia & 16253,8 & 28755,8 & 1,1 \\
19 & Suecia & 8843,5 & 26453,7 & 1,0 \\
20 & Brasil & 15943,3 & 25409,8 & 1,0 \\
21 & Venezuela & 16857,6 & 24598,7 & 1,0 \\
\hline
\end{tabular}

Fuente: OEEE - Ministerio de Agricultura. 


\section{El comportamiento de la agricultura en la crisis internacional. \\ Resultados a fines del 2009}

La crisis internacional nos demostró la problemática a la que se enfrentó el sector agrícola de nuestro país durante todo este breve periodo, que para algunos importantes investigadores no ha concluido, por lo cual habría que estar alerta de los principales compradores de nuestros productos. Al medir el aporte de la agricultura al PBI nacional nos damos cuenta que su valor relativo a disminuido en el período 2007-2009. Pero lo que no significa que su aporte al PBI ha disminuido en la economía internacional tal como hemos explicado anteriormente. Ocurre que el aporte relativo del sector construcción ha crecido significativamente.

Cuadro 7. La producción agrícola en el PBI.

\begin{tabular}{|l|c|c|c|c|c|}
\hline & 2005 & 2006 & 2007 & 2008 & 2009 \\
\hline Agropecuario 2/ & 8,25 & 8,30 & 7,87 & 7,68 & 7,79 \\
Agrícola & 5,10 & 5,13 & 4,0 & 4,70 & 4,70 \\
Pecuario & 2,47 & 2,48 & 2,40 & 2,31 & 2,40 \\
Pesca & 0,54 & 0,51 & 0,50 & 0,49 & 0,45 \\
Minería e & 6,59 & 6,20 & 5,85 & 5,73 & 5,72 \\
hidrocarburos & 15,40 & 15,37 & 15,67 & 15,56 & 14,33 \\
Manufactura & 2,08 & 2,07 & 2,06 & 2,02 & 2,024 \\
Electricidad y agua & 4,90 & 5,21 & 5,58 & 5,92 & 6,23 \\
Construcción & 14,01 & 14,52 & 14,62 & 15,04 & 14,85 \\
Comercio & 48,24 & 47,83 & 47,86 & 47,55 & 48,61 \\
Otros servicios 3/ & 100,00 & 100,00 & 100,00 & 100,00 & 100,00 \\
\hline PBI
\end{tabular}

Fuente: Banco Central de Reserva del Perú - Memoria Institucional 2009.

\section{COMENTARIOS FINALES}

1. La reciente crisis pone en evidencia que la globalización a la par que genera oportunidades para países como el Perú también genera riesgos que deben ser superados de una manera rápida. Hoy en día, las actividades comerciales y financieras exigen una mayor cooperación mundial, reconociendo esto como una meta de largo esfuerzo ya que se observa un comportamiento dominante en los países que fueron grandes actores de la economía mundial y también en los nuevos. 


\section{Pedro Barrientos Felipa}

2. Las noticias que se tienen del mercado internacional es que la vulnerabilidad del sistema financiero aún persiste y afecta la relación entre organizaciones y países, y por lo tanto en su comercialización. Las expectativas acerca de lo que puede ocurrir y afectar a determinados sectores económicos subsisten y debe tomarse decisiones con sumo cuidado, más aún en países que como el nuestro están emergiendo y buscan un lugar importante en la comercialización de productos agrícolas, sean estos commodities o nuevos productos. Además, debe considerarse que hay un grado de dependencia relativamente alto de importaciones agrícolas.

3. La crisis mundial lleva a los empresarios - de cualquier lugar del mundo- a continuar haciendo ajustes en la productividad, considerando que el riesgo puede ser minimizado con la disminución de los costos de sus productos agrícolas de exportación. Cada país tiene respuestas diferentes y todos los países de alguna manera están conectados. La competencia se hará más intensa, y los compradores se harán más exigentes en sus estrategias de comercialización. No se puede decir que ya se superó el problema de la crisis, hay que estar alertas y preparados ante el panorama que se presenta.

4. Las exportaciones agrícolas tienen un mercado potencial significativo, pudiendo aprovechar la existencia de segmentos de mercado que no son adecuadamente atendidos. Sin embargo, debe considerarse el concepto de "valor agregado", así como el concepto de "valor del producto". Ahí tenemos el caso de China e India, nuevos mercados que son socios comerciales de nuestro país pero que falta desarrollarlos. Incluso puede ser el caso de ingresar a mercados sofisticados como es el de productos ecológicos en mercados (ciudades) en que sus ciudadanos están mejorando su nivel de ingreso familiar.

5. Lo anterior explica que en nuestro país la agricultura de exportación tiene posibilidades significativas, y aquí hay que incluir a la agricultura de mediana y pequeña dimensión. Es una manera que puede ayudar a generar ingresos a los pequeños agricultores. Puede hacerse uso de modelos de clusterización. La agricultura peruana en el período de crisis ha crecido.

6. Estar en la búsqueda de soluciones de corto plazo, no debe dejarnos de tomar en 


\section{El comportamiento de la agricultura en la crisis internacional. Resultados a fines del 2009}

cuenta lo que debe ocurrir en el largo plazo. La visión debe llevarnos a cambiar patrones estructurales que llevan al país a presentar vulnerabilidades. La mejora de la producción o productividad en el agro debe estar relacionado a cambios en el sistema educativo y sanitario, así como el de la mejora de infraestructura. Todo esto con la finalidad de pasar a un estadio en que la agricultura tenga una producción con mayor valor agregado.

\section{BIBLIOGRAFÍA}

Arroyo, Eduardo (2009). Crisis financiera y sus repercusiones en el Perú, p. 17.

Asociación Nacional de la Empresa Privada (2008). Crisis financiera y escalada de precios de materias primas: impactos sobre América Latina, p. 6.

Conferencia de las Naciones Unidas sobre Comercio y Desarrollo - UNCTAD (2009). La crisis económica mundial: fallos sistémicos y remedios multilaterales. Nueva York. p. 19.

Cuadrado Roura, Juan (2000). Política económica: objetivos e instrumentos. 2. ${ }^{\text {a }}$ ed. Madrid: McGraw Hill Interamericana de España.

Ginesta, Jacques (2009). La actual crisis económica y algunas de sus consecuencias políticas. p. 28.

Instituto Interamericano para la Cooperación en la Agricultura (2009). Informe 2008: Perú. La contribución del IICA al desarrollo de la agricultura y las comunidades rurales. IICA. p. 36

(2009). Agricultura de América Latina y el Caribe: bastión ante la crisis mundial y motor para el desarrollo futuro. IICA. p. 30

Organización de las Naciones Unidades para la Agricultura y la Alimentación (2009). 


\section{Pedro Barrientos Felipa}

